\title{
Direct Observation of Magnetostatic Coupling of Chain Arrays of Magnetic Disks
}

\author{
Xiaobin Zhu, Vitali Metlushko, Bojan Ilic, and Peter Grutter
}

\begin{abstract}
Magnetostatic coupling of Permalloy disk chains were studied by magnetic-force microscopy. The annihilation process occurs at the center of the chain, while nucleation initiates at the edge of the chain. The switching is strongly coupled along the chain direction.
\end{abstract}

Index Terms-Magnetic-force microscopy (MFM), magnetostatic coupling, vortex annihilation, vortex nucleation.

\section{INTRODUCTION}

$\mathbf{C}$ IRCULARLY shaped magnetically soft particles of submicrometer size can form vortex structures with a possible vortex core singularity in the center. When these particles are placed very close to each other, they are magnetostatically coupled to each other.

Recent experiments by magnetooptical Kerr-effect measurements [3], [4], Brillouin light-scattering spectroscopy [5], and magnetic-force microscopy (MFM) [6] indicate that interdot coupling can modify the magnetic properties of magnetic dots such as the switching field [3]-[6] and anisotropy [5], [6]. The coupling originates from magnetic dipole and higher order multipole interactions.

The purpose of this study is to directly visualize the magnetostatic coupling in truncated chains of Permalloy disks.

\section{EXPERIMENTAL METHODS}

\section{A. Sample Preparation}

Separated Permalloy disks and chains of disks were prepared by a standard electron-beam lithography technique followed by liftoff. The electron-beam writer is a modified JEOL 840 scanning electron microscope system, and the magnetic thin film was deposited using an electron-beam evaporator with a base pressure of $10^{-9}$ torr. Fig. 1 shows scanning electron microscopy images of Permalloy arrays with diameter of $500 \mathrm{~nm}$ and a thickness of $40 \mathrm{~nm}$.

Manuscript received January 9, 2003. This work was supported in part by Le Fonds Pour la Formation de Chercheurs et I'Aide a la Recherche de la Province de Quebec, by the Natural Sciences and Engineering Research Council of Canada, and by the Canadian Institute for Advanced Research.

X. Zhu was with the Department of Physics, McGill University, Montreal, QC H3A 2T8, Canada. He is now with the Department of Physics, University of Alberta, Edmonton, AB T6G 2J1, Canada (e-mail: xbzhu @ physics.mcgill.ca; xzhu@phys.ualberta.ca).

V. Metlushko is with the Department of Electrical and Computer Engineering, University of Illinois at Chicago, Chicago, IL 60607-0024 USA (e-mail: vmetlush@ece.uic.edu).

B. Ilic is with the School of Applied and Engineering Physics, Cornell University, Ithaca, NY 14853 USA.

P. Grütter is with the Department of Physics, McGill University, Montreal, QC H3A 2T8, Canada (e-mail: grutter@ physics.mcgill.ca).

Digital Object Identifier 10.1109/TMAG.2003.815586
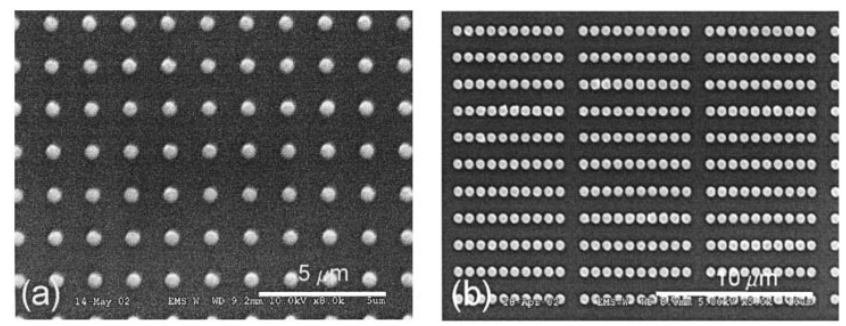

Fig. 1. Scanning electron microscopy images of 40-nm-thick Permalloy disk samples with a diameter of $500 \mathrm{~nm}$. (a) Array of individual disks separated by $1.5 \mu \mathrm{m}$. (b) Truncated chains with disk spacing of $100 \mathrm{~nm}$.
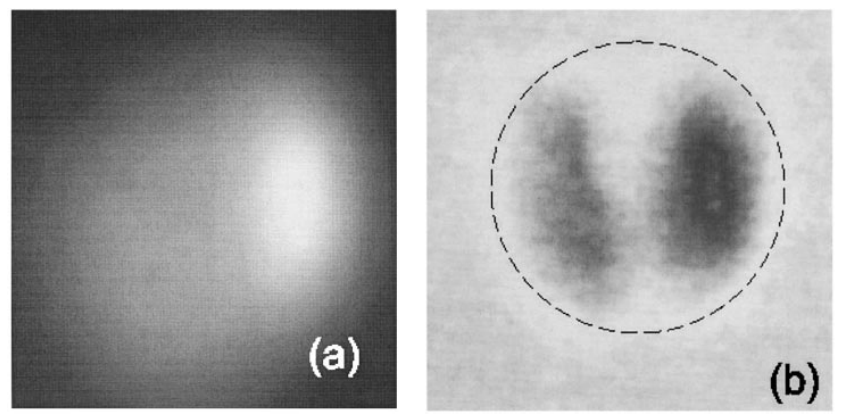

Fig. 2. (a) Topography and (b) MFM image of a Permalloy disk with a size of $500 \mathrm{~nm}$ and a thickness of $40 \mathrm{~nm}$.

\section{B. Experimental Techniques}

The MFM experiments were studied with a custom-built system in moderate vacuum of $1 \times 10^{-5}$ torr. Silicon cantilevers sputter coated with $20-\mathrm{nm} \mathrm{Co} \mathrm{Co}_{71} \mathrm{Pt}_{12} \mathrm{Cr}_{17}$ were used as force sensors. The experiments were performed in the constant height mode to reduce MFM tip stray field induced irreversible distortions to the sample magnetization [7].

\section{RESULTS AND DISCUSSION}

\section{A. Switching of Individual Permalloy Disks}

Magnetic vortex structures are energetically stable in submicrometer-sized magnetic particles and have been observed by various techniques such as MFM [6], [8], spin polarized scanning tunneling microscopy [9], and Lorentz microscopy [10]. Fig. 2(a) and (b) shows a typical disk and its magnetic contrast. As can be seen clearly, the disk forms a vortex structure.

A distinct characteristic of magnetic disks is that the magnetization reversal has two transitions: a nucleation and an annihilation of the magnetic vortex. The switching process can be described as follows. Starting from the vortex state, if an in-plane magnetic field is applied, the vortex will be distorted. The vortex core will move closer to the disk edge perpendicular to the field 

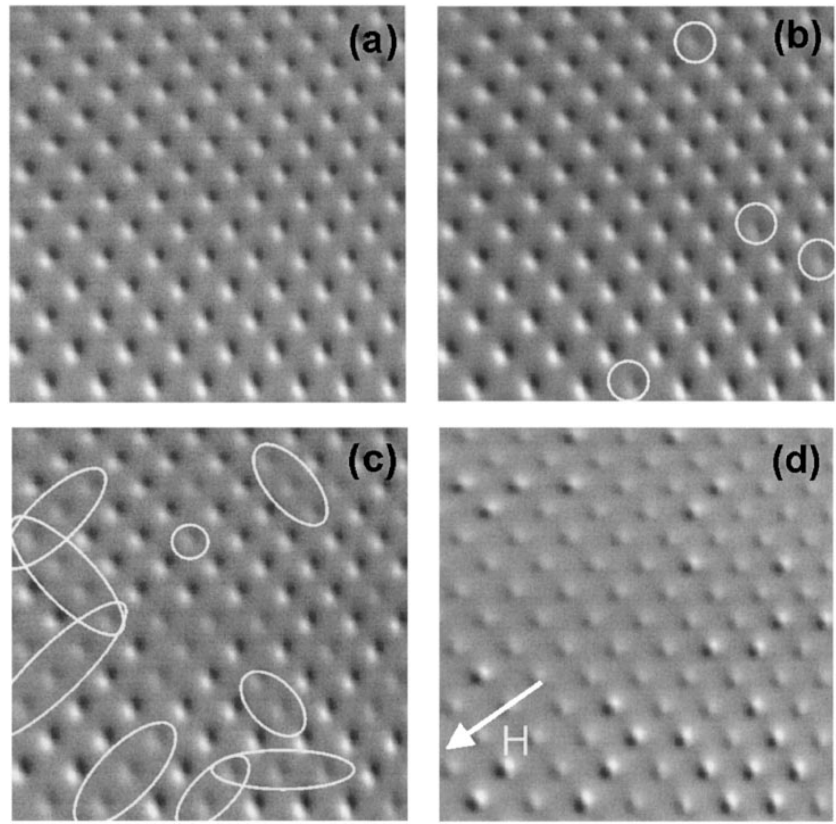

Fig. 3. MFM image after different magnetic fields are applied. (a) 650 Oe (b) 145 Oe. (c) 120 Oe. (d) - 500 Oe. Images (a)-(c) were imaged at magnetic field of 180 Oe and (d) was imaged at -180 Oe. The arrow in (d) indicates the field direction. Some switched disks are circled in (b) and (c). Scan size: $20 \mu \mathrm{m}$.

direction. If the magnetic field is large enough $(H>H a)$, the vortex will be expelled and the disk will form a single-domain state. This is also known as the "annihilation process" [3], [6]. However, if we start from the single-domain state, and the magnetic field is reduced below a critical value, the nucleation field $H n$, Zeeman energy, and exchange energy cannot overcome the demagnetization energy. The vortex is then created in the dot—the "nucleation process" [3], [6].

It is challenging to obtain the precise nucleation field $H n$ and annihilation field $\mathrm{Ha}$ by MFM, as the tip stray field can switch the particle during imaging in the presence of an external magnetic field [7]. To eliminate this, the MFM experiments need to be performed at an external field $H$ with $H n<H<H a$ after the field is ramped to a designated value. This allows the precise switching field to be obtained [6].

Fig. 3(a)-(d) shows the MFM images after different magnetic fields are applied. Fig. 3(a) shows that all the disks form a single-domain state after application of 650 Oe, much larger than $\mathrm{Ha}$. If the applied field is reduced to $145 \mathrm{Oe}$, Fig. 3(b) shows some elements have formed a vortex state, as indicated by white circles. Note that particles in the vortex state still show a weak bipolar contrast. This is because the image is taken in the presence of a magnetic field of $\mathrm{H}=180 \mathrm{Oe}$ instead of at remanence, as $\mathrm{H}=180$ Oe fulfills $H n<H<H a$ for all disks in the sample, thus avoiding tip-induced transitions. As the magnetic field is further reduced, more and more disks form vortex states. Fig. 3(c) shows the image when the field is reduced to $12 \mathrm{O}$ Oe. As the inter-disk separation is large, the inter-disk coupling is a negligible contribution to the switching field of each individual dot. The switching field variation among different particles with a switching field ranging from 55 to $155 \mathrm{Oe}$, therefore, comes from individual dot differences.
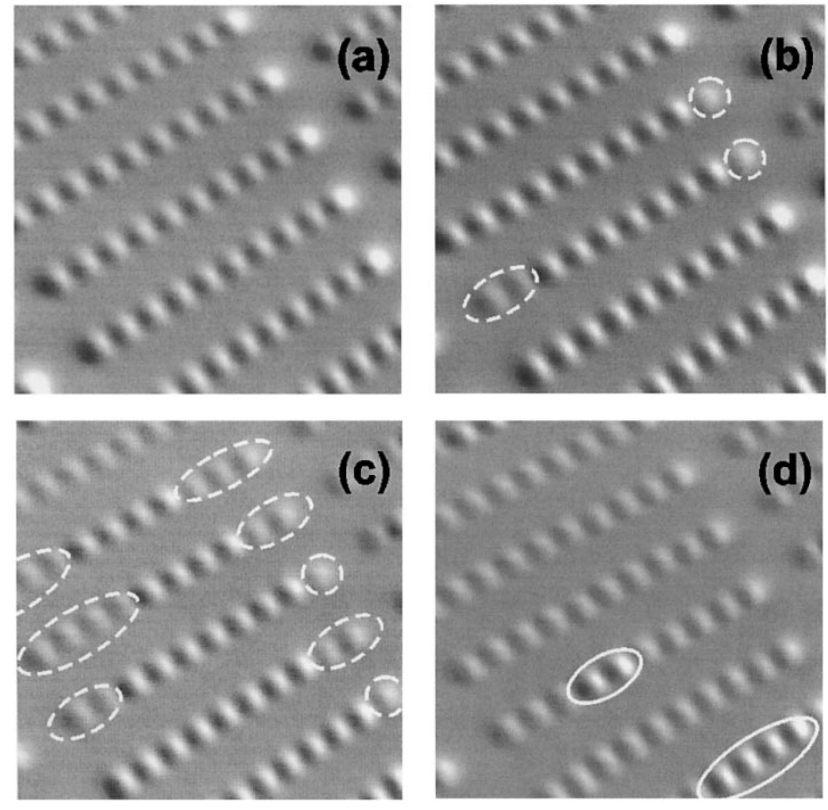

Fig. 4. Images of Permalloy disk chains after applying magnetic field of: (a) 800 , (b) 195 , (c) 162, and (d) 146 Oe. Images were taken at magnetic field of 250 Oe. The field is applied along the chains. Disks switched into vortex states are circled in (b) and (c). Disks switched into single-domain states are circled in (d). Scan area: $8 \mu \mathrm{m}$.

If a large reversed field is applied, the vortex structure is switched to the reversed single-domain state. Fig. 3(d) shows the image after -500 Oe is applied.

\section{B. Switching of Permalloy Disk Array}

When the disks are placed very close to each other, as in Fig. 1(b), smaller than the Permalloy disk size, these Permalloy disks are then magnetostatic coupled. The coupling will have a strong influence on the switching behavior, switching field, and initial susceptibility of the disk arrays [10].

Fig. 4 shows the MFM data of nucleation of the Permalloy disk chains of Fig. 1(b). If a large magnetic field is applied, all the disks form a single-domain state, as shown in Fig. 4(a). The MFM contrast at the edges of each chain is brighter or darker than the disks inside the chains, as the stray field of neighboring disks tend to cancel each other. However, if the magnetic field is reduced to a certain value, some disks in some chains will be switched to the vortex state. Fig. 4(b) shows an MFM image at 250 Oe after the magnetic field is reduced to $195 \mathrm{Oe}$. As can be seen, four disks (circled in this figure) have formed vortex states resulting in a much weaker contrast. As the magnetic field is further reduced, more disks form coupled vortex structures forming chain structures, as can be seen in Fig. 4(c). This process is initiated at the edge of the chain, and then propagates toward the center of the chains until all the chains form a vortex state, as can be seen in Fig. 4(d). Note that, in Fig. 4(d), several chains have completely transformed to the vortex state, while a few disks have not yet been switched, as indicated by the solid circles.

This is direct evidence that the Permalloy disks are magnetostatically coupled. The observed results are consistent with micromagnetic simulation proposed by Novosad et al. [11], in which the demagnetization curves for the disk chains with small 

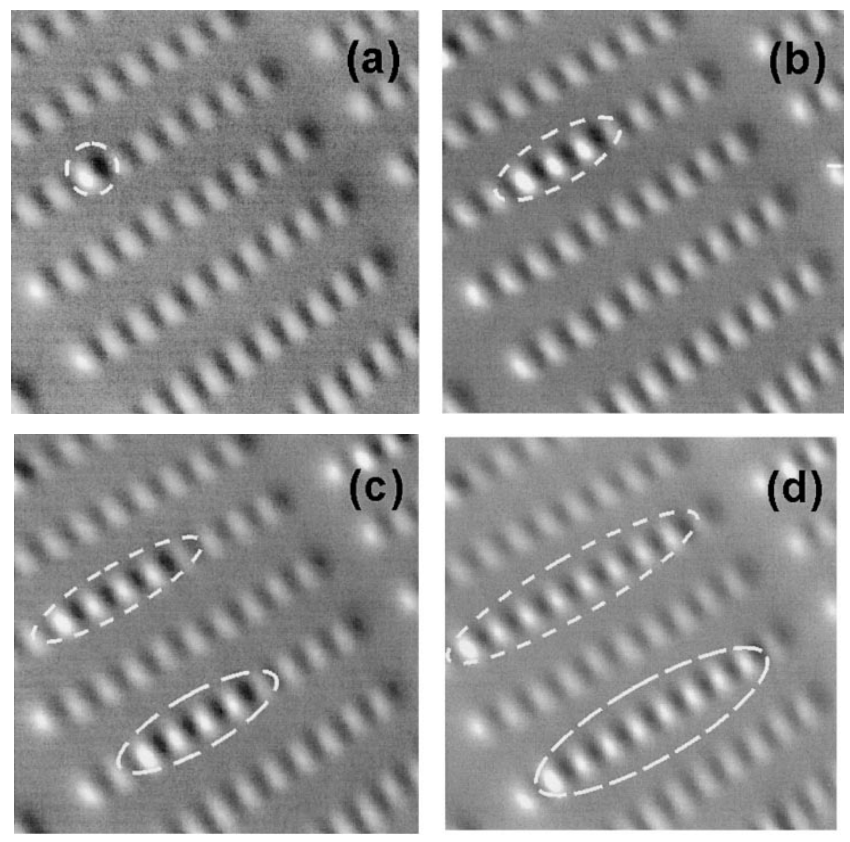

Fig. 5. Images of Permalloy disk chains after applying magnetic field of: (a) 425, (b) 440, (c) 455, and (d) 489 Oe. Images were taken at a magnetic field of 250 Oe. The switched disks are circled in (a)-(d). Scan size: $8 \mu \mathrm{m}$.

interdot distance exhibit a step-wise decrease. The vortex nucleation is first initiated in the two dots located at the ends of the chains. This is because these dots have fewer neighboring particles resulting in a smaller effective magnetic field. Once the disks at the edge nucleate a vortex state, their nearest neighbor disks are subsequently exposed to a reduced effective field, and the nearest neighbor disks can more easily nucleate a vortex. We find that the nucleation process for different chains does not occur at the same magnetic field. This is due to switching field variation of different disks.

Magnetostatic coupling can also be demonstrated in the annihilation process of the chains. The annihilation process is initiated at the central disks of the chains, as these central disks are exposed to the largest effective magnetic field produced by their neighbors. Fig. 5 shows this process. For small fields of $425 \mathrm{Oe}$, one of the disks in a chain forms a single-domain state in Fig. 5(a). As a larger field 440 Oe is applied, more vortices along the chain are annihilated, as shown in Fig. 5(b). Fig. 5(c) shows the images at even larger fields. Switched dots themselves will produce a larger effective field. Therefore, the neighboring disks are then more easily switched to the single-domain state. This process propagates toward the end of the chains within a small field range. Fig. 5(d) shows all the elements in two chains almost forming a single-domain state. It needs to be pointed out that the switching field for different chain varies a lot. Note in Fig. 5(d), although some chains are almost completely switched, there are other chains where no particle has switched. This is a direct consequence of coupling among the particles in a given chain.

The observed phenomena can be explained by an effective field produced by neighboring dots. This coupling is rather complicated when the dots are placed very close to each other, as higher order multipolar interactions need to be indicated. Micromagnetic simulations of a Permalloy chain structure are currently in process.

\section{CONCLUSION}

Permalloy disks of 500-nm diameter and 40-nm thickness and chains of disks with spacing of $100 \mathrm{~nm}$ were studied by MFM. MFM indicates that individual Permalloy disk switching is the result of vortex nucleation and annihilation. Permalloy disks in truncated chains are strongly coupled. The nucleation process is initiated at the ends of the chains, while annihilation first happens centrally in the chains.

\section{REFERENCES}

[1] R. P. Cowburn and M. E. Welland, "Room temperature magnetic quantum cellular automata," Science, vol. 287, pp. 1466-1468, Feb. 2000.

[2] R. P. Cowburn, "Property variation with shape in magnetic nanoelements," J. Phys. D, Appl. Phys., vol. 33, pp. R1-R16, Jan. 2000.

[3] V. Novosad, K. Yu. Guslienko, H. Shima, Y. Otani, S. G. Kim, K. Fukamichi, N. Kikuchi, O. Kitakami, and Y. Shimada, "Effect of interdot magnetostatic interaction on magnetization reversal in circular dot arrays," Phys. Rev. B, Condens. Matter, vol. 65, pp. 060 402-060 405, Feb. 2002.

[4] M. Natali, A. Lebib, Y. Chen, I. L. Prejbeanu, and K. Ounadjela, "Configurational anisotropy in square lattices of interacting cobalt dots," $J$. Appl. Phys., vol. 91, pp. 7041-7043, May 2002.

[5] C. Mathieu, C. Hartmann, M. Bauer, O. Buettner, S. Riedling, B. Roos, S. O. Demokritov, B. Hillebrands, B. Bartenlian, C. Chappert, D. Decanini, F. Rousseaux, E. Cambril, A. Müller, B. Hoffmann, and U. Hartmann, "Magnetic coupling of Permalloy micron dots forming a square lattice," Appl. Phys. Lett., vol. 70, pp. 2912-2914, May 1997.

[6] X. Zhu, V. Metlushko, B. Ilic, and P. Grütter, "Magnetization reversal and configuration anisotropy of closely packed Permalloy dot arrays," Appl. Phys. Lett., vol. 80, pp. 4789-4791, June 2002.

[7] X. Zhu, V. Metlushko, B. Ilic, and P. Grütter, "Magnetic force microscopy study of electron-beam-patterned soft Permalloy particles: Technique and magnetization behavior," Phys. Rev. B, Condens. Matter, vol. 66, pp. 024 423-024 440, July 2002.

[8] T. Shinjo, T. Okuno, R. Hassdorf, K. Shigeto, and T. Ono, "Magnetic vortex core observation in circular dots of Permalloy," Science, vol. 289, pp. 930-932, Aug. 2000.

[9] A. Wachowiak, J. Wiebe, M. Bode, O. Pietzsch, M. Morgenstern, and R. Wiesendanger, "Direct observation of internal spin structure of magnetic vortex cores," Science, vol. 298, pp. 577-580, Oct. 2002.

[10] M. Schneider, H. Hoffman, and J. Zweck, "Lorentz microscopy of circular ferromagnetic Permalloy nanodisks," Appl. Phys. Lett., vol. 77, pp. 2909-2911, Oct. 2000

[11] V. Novosad, K. Yu. Guslienko, Y. Otani, H. Shima, and K. Fukamichi, "Magnetostatic interdot dot coupling in arrays of circular ferromagnetic dots," J. Magn. Magn. Mater, vol. 239, pp. 234-236, Feb. 2002. 